\title{
A Current-Mode FDNR Circuit Element Using Capacitive Gyrators
}

\author{
Vladimir I. Prodanov \& Michael M. Green \\ Department of Electrical Engineering \\ College of Engineering and Applied Sciences \\ State University of New York at Stony Brook \\ Stony Brook, NY 11794-2350
}

\begin{abstract}
A current-mode implementation of a frequency-dependent negative resistance (FDNR) element is presented using a novel capacitive gyrator. This FDNR element lends itself well to the design of low-pass ladder filters and its use will result in a more efficient integrated circuit implementation than filters that simulate floating inductors using resistive gyrators.
\end{abstract}

\section{INTRODUCION}

It is well known that analog filters based on LC ladder networks exhibit very low sensitivity with respect to component variations in both the passband and the stopband [1]. A number of techniques have been developed that allow design of these filters without requiring the use of inductors which, in the design of discrete filters, tend to be bulky and have unfavorable characteristics, and, in the case of monolithic filters, simply cannot be used.

The most common technique presently used is the connection of a gyrator and a capacitor to realize a oneport or two-port that behaves like a grounded or floating inductor. The current-mode realization of a gyrator shown in Fig. 1 has a number of desirable characteristics for IC design including low power, small chip area and tunability [2]. A circuit that behaves like a grounded inductor using a current-mode realization of the gyrator is shown in Fig. 1a. The floating inductor realization, shown in Fig. $1 b$, requires twice as many transconductance blocks. This is a disadvantage for the design of active low-pass ladder networks since series inductors are always required. Nontheless, filters using these structures have been successfully designed and fabricated [3].

Another approach to designing ladder filters without inductors has been to multiply the impedance of each

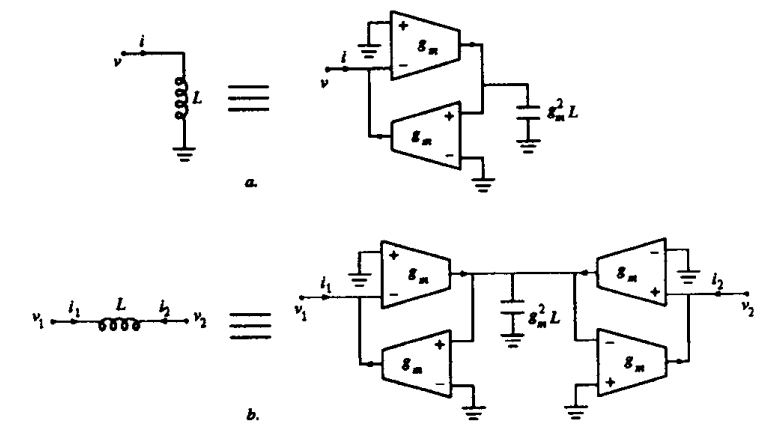

Figure 1: Active Realizations of (a.) Grounded and (b.) Floating Inductors

two-terminal element in the passive prototype filter by $k / s$, where $k$ is a scaling factor. This transformation results in the element substitutions shown in Fig. 2. The element that results from performing this substitution on a capacitor is known as a frequency-dependent negative resistance (FDNR) element. Fig. 3 illustrates the transformation on a low-pass filter structure. The large-valued resistors $R_{S}^{\prime}$ and $R_{L}^{\prime}$ placed in parallel with the terminating capacitors are required for proper low-frequency response ${ }^{1}$. The advantage of the FDNR approach is that all active elements in this circuit are grounded. However, the most common FDNR active realization, shown in Fig. 5, requires two voltage-mode op-amps and is not easily tunable; hence, this approach does not lend itself well to a monolithic realization.

In this paper we present a new two-port element called a capacitive gyrator and show how it can be used

\footnotetext{
${ }^{1}$ An alternative to this requirement is to begin with a singlyterminated ladder filter which results, after the transformation, in a circuit with a dc path from the input to the output [4]. However singly-terminated ladder filters do mot have the same desirable tolerance to component variations as the doubly-terminated filters [1]
} 


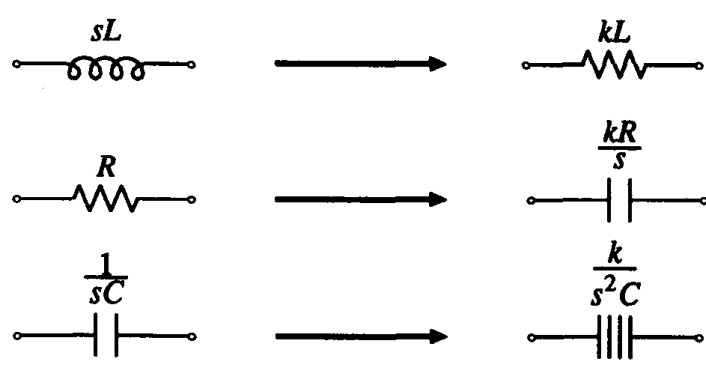

Figure 2: Element Substitutions for FDNR Realization

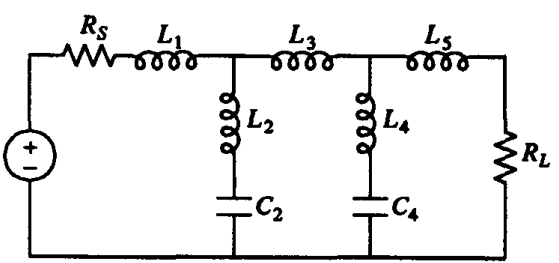

a.

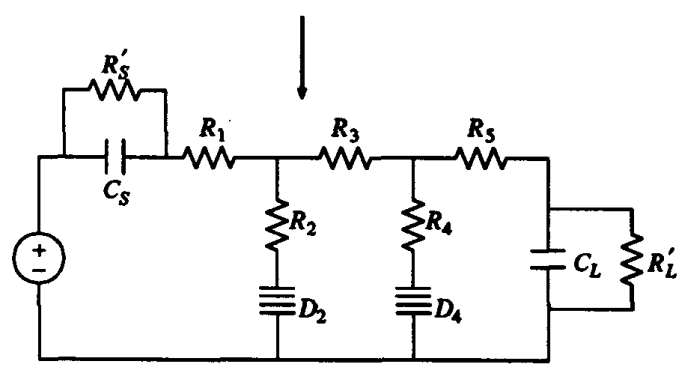

b.

Figure 3: Transformation of Low-Pass Filter for FDNR Realization

to construct a compact current-mode grounded FDNR element that can be made tunable. An elliptic low-pass ladder filter is used as an example.

\section{NEW CIRCUIT ELEMENTS}

Fig. $6 a$ shows a current-mode two-port that behaves as a capacitive gyrator. Its operation is identical to a standard resistive gyrator except that the gyration resistance has been replaced by a gyration capacitance. The conductance matrix for this two-port is given by

$$
\left(\begin{array}{c}
i_{1} \\
i_{2}
\end{array}\right)=\left[\begin{array}{cc}
0 & s C \\
-s C & 0
\end{array}\right]\left(\begin{array}{l}
v_{1} \\
v_{2}
\end{array}\right)
$$

It can easily be shown that if port 2 is terminated by an impedance $Z_{L}$, then the impedance $Z_{1}$ looking into port

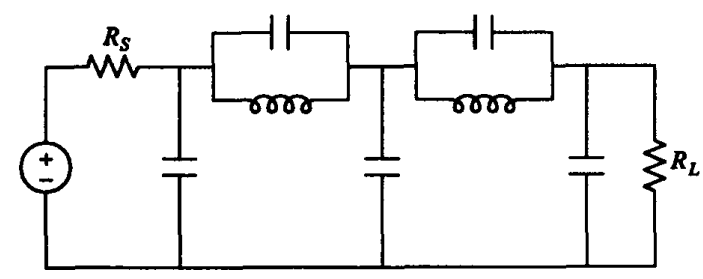

Figure 4: Minimum Inductor Low-Pass Structure

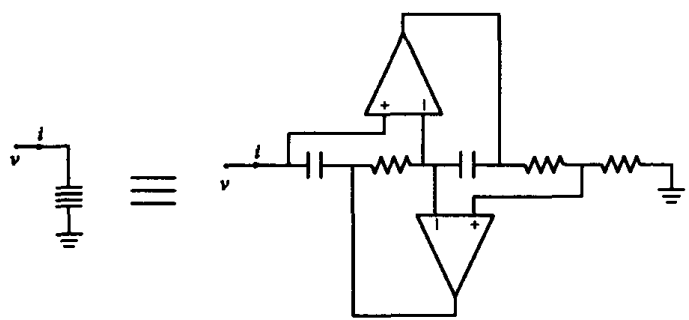

Figure 5: Conventional Active Realization of FDNR Element

1 is given by

$$
Z_{1}=\frac{1}{s^{2} C^{2} Z_{L}}
$$

If we make $Z_{L}$ a resistor as shown in Fig. $6 b$, then the resulting one-port behaves like a grounded FDNR. Notice that this circuit uses the same number of active transimpedance blocks as the realization of a grounded inductor shown in Fig. $1 a$ and half the number of active transimpedance blocks as the realization of a floating inductor shown in Fig. $1 b$. Comparing the Fig. $3 b$ filter, which uses two grounded FDNR elements (realized by four transcapacitance blocks), with the Fig. 4 filter which uses two floating inductors, (realized by eight transconductance blocks ${ }^{2}$ ), we see that in general, low-pass ladder filters using the FDNR elements shown in Fig. 6 result in circuits with less chip area and less power dissipation than filters using floating gyrator inductors.

A simple BiCMOS implementation of the capacitive transconductance block, where $i_{\text {out }}=s C\left(V_{+}-V-\right)$, is shown in Fig. $6 c$. A tunable realization using a pchannel differential pair and a Gilbert gain cell is shown in Fig. 6d. The transfer characteristic for this circuit is given by

$$
i_{\text {out }}=\left(1+\frac{I_{x}}{I_{y}}\right) s C V_{\text {in }} .
$$

Hence all transcapacitances in a filter can be tuned uniformly by varying the ratio $I_{x} / I_{y}$.

\footnotetext{
${ }^{2}$ Tunable active realizations of both circuits would require active blocks for the input and output terminating elements as well, resulting in six transcapacitance blocks and ten transconductance blocks, respectively.
} 


\section{FILTER EXAMPLE}

The fifth-order elliptic filter based on Fig. $3 b$ and shown in Fig. $7 a$ was designed and simulated using the models from the MOSIS 2-micron ORBIT BiCMOS process. The transcapacitance block shown in Fig. $6 c$ was used. Deviations from ideal behavior in the active circuit, illustrated in Fig. 8, come from the $r_{e}$ effectively in series with the capacitor in the Fig. $6 c$ transcapacitance block which results in added loss in the FDNR element.

\section{CONCLUSIONS}

We have presented a new circuit element called a capacitive gyrator and have shown that it can be used to make FDNR elements suitable for making tunable integrated low-pass ladder filters. A floating inductor realization requires four transconductance blocks and a capacitor; a grounded FDNR requires only two transconductance blocks and two capacitors. Hence the filter design techniques presented in this paper provide a suitable method for realizing efficient analog integrated low-pass filters.

Research is now under way on an improved tunable transcapacitance block based on the circuit shown in Fig. $6 d$. A fully tunable version of the filter shown in Fig. $7 b$ is currently being designed and will be tested in Spring 1994.

\section{REFERENCES}

[1] G. C. Temes and J. W. LaPatra, Introduction to Circuit Synthesis and Design. New York: McGrawHill, 1977, Ch. 6.

[2] A. S. Sedra and P. Prackett, Filter Theory and Design: Active and Passive. Forest Grove, OR: Matrix Publishers.

[3] C. Toumazou, F. J. Lidgey and D. G. Haigh, Analog IC Design: The Current-Mode Approach. London: Peregrinus Ltd., Ch. 5.

[4] F. Krummenacher and N. Joehl, “A 4-MHz CMOS continuous-time filter with on-chip automatic tuning," IEEE Journal on Solid-State Circuits, vol. 23, June 1988, pp. 751-758.

[5] J. Hutchison and F. F. Lee, "Some notes on practive FDNR filters," IEEE Transactions on Circuits and Systems, vol. 28, pp. 242-245, March 1981.
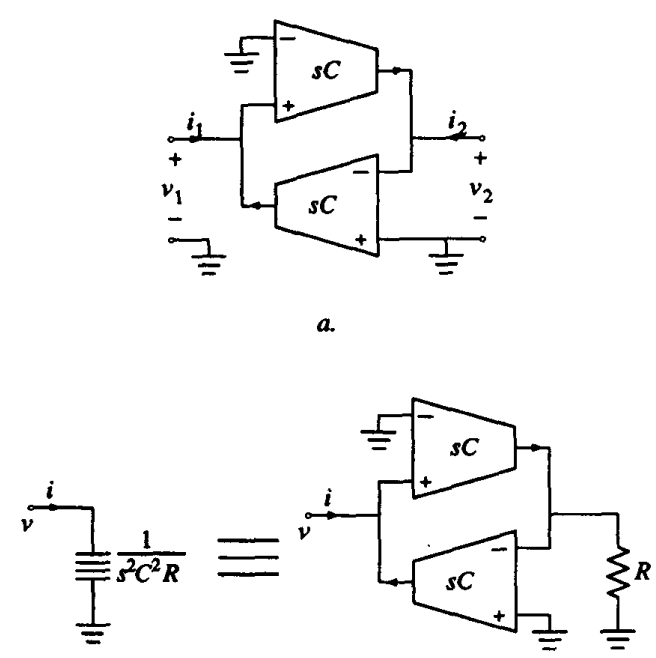

b.

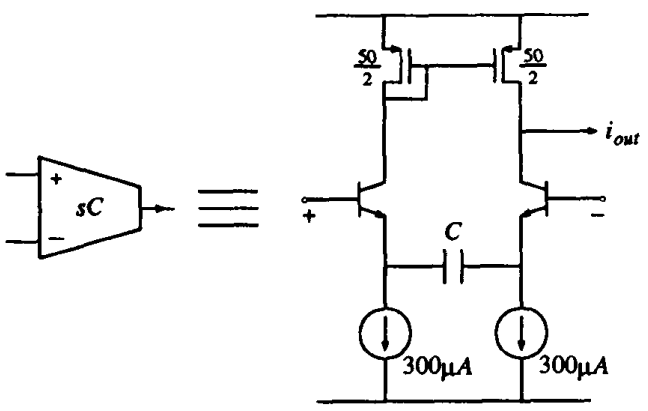

c

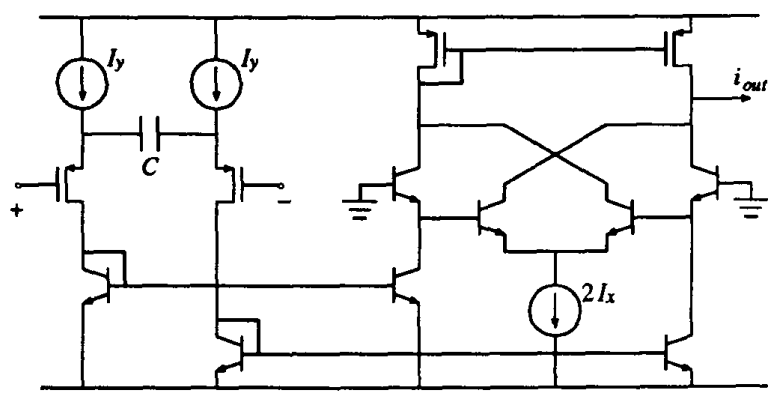

d.

Figure 6: $a$. Current-Mode Capacitive Gyrator; $b$. FDNR Element Realization; $c$. Simple Transcapacitive Block; d. Tunable Transcapacitive Block 

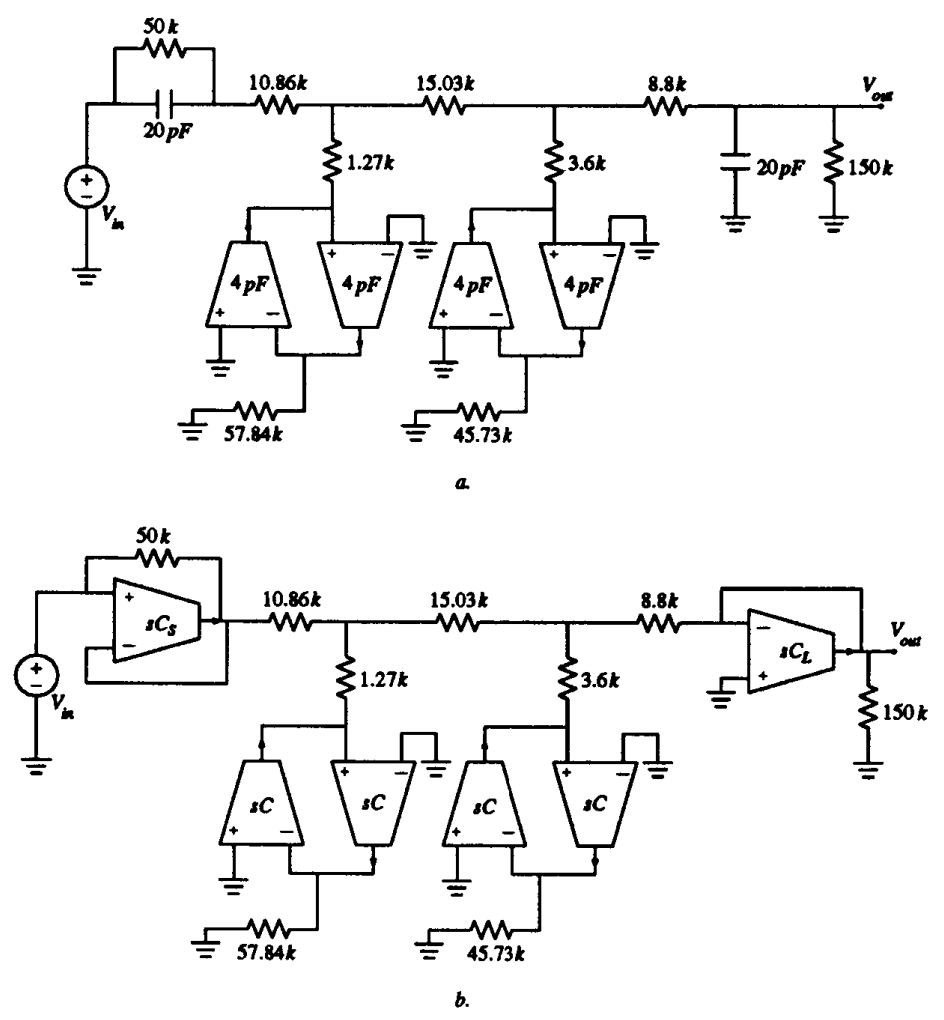

Figure 7: Fifth-Order Elliptic Filter: (a.) Fixed-Frequency Version; (b.) Tunable Version

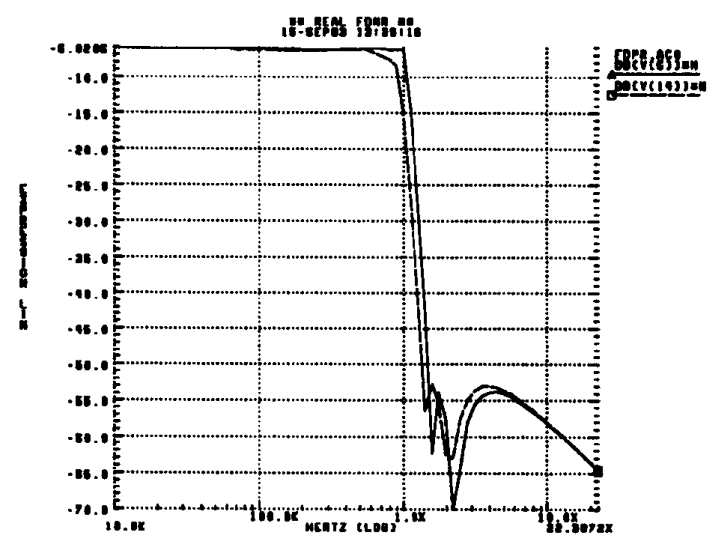

Figure 8: Magnitude vs. Frequency Plot of Passive Prototype Filter (Solid Line) and Active FDNR Filter (Dashed Line) 